\title{
Le déficit de l'attention / hyperactivité (TDA/H) et les comportements violents des jeunes en milieu scolaire
} L'état de la question

\section{Attention deficit disorder / hyperactivity (add/H) and the} violent behaviour of youth in the school setting The current situation

\section{El déficit de atención / hiperactividad (tda/h) y los comportamientos violentos de los jóvenes en el medio escolar El estado de la cuestión}

\section{Josée Juneau et Louis-Philippe Boucher}

Volume 32, numéro 1, printemps 2004

La violence en milieu scolaire

URI : https://id.erudit.org/iderudit/1079114ar

DOI : https://doi.org/10.7202/1079114ar

\section{Aller au sommaire du numéro}

\section{Éditeur(s)}

Association canadienne d'éducation de langue française

\section{ISSN}

0849-1089 (imprimé)

1916-8659 (numérique)

\section{Découvrir la revue}

\section{Citer cet article}

Juneau, J. \& Boucher, L.-P. (2004). Le déficit de l'attention / hyperactivité (TDA/H) et les comportements violents des jeunes en milieu scolaire : l'état de la question. Éducation et francophonie, 32(1), 38-53.

https://doi.org/10.7202/1079114ar
Résumé de l'article

Cet article vise, d'une part, à faire le point sur l'état des connaissances actuelles en regard de ce qu'il est convenu d'appeler le trouble relatif au déficit de l'attention avec ou sans hyperactivité (TDA/H), et d'autre part, à examiner dans quelle mesure les élèves qui en sont atteints manifestent des comportements violents et s'il existe des types d'intervention aptes à contribuer à les réduire. Bien qu'il soit le trouble de comportement le plus commun diagnostiqué au cours de l'enfance, le TDA/H est plutôt mal connu et difficile à identifier et à diagnostiquer. Compte tenu de l'ampleur du phénomène, des conséquences observées sur le comportement des élèves qui en sont atteints, il s'avère important d'en connaître les symptômes et les conséquences, plus particulièrement en ce qui concerne les comportements violents. Les études tendent à montrer que les élèves ayant un TDA/H sont significativement plus nombreux que les autres à manifester des comportements colériques, agressifs, voire violents envers leurs pairs. De tels comportements entraînent des situations de conflit, d'impopularité et de rejet de la part de leurs pairs et plus d'attention négative de leur enseignant. Les interventions qui font appel à des moyens qui aident les individus à contrôler leurs comportements apparaissent particulièrement appropriées pour amener les jeunes atteints de ce syndrome à réduire leurs comportements agressifs et violents et à développer leurs compétences sociales.

Tous droits réservés $@$ Association canadienne d’éducation de langue française, Ce document est protégé par la loi sur le droit d’auteur. L’utilisation des 2004 services d’Érudit (y compris la reproduction) est assujettie à sa politique d'utilisation que vous pouvez consulter en ligne.

https://apropos.erudit.org/fr/usagers/politique-dutilisation/ 


\section{Le déficit de l'attention / hyperactivité (TDA/H) et les comportements violents des jeunes en milieu scolaire : l'état de la question}

Josée Juneau, Ph. D

Faculté des sciences de l'éducation, Université Laval, Canada

Louis-Philippe Boucher, Ph. D

Département des sciences de l'éducation, Université du Québec à Chicoutimi, Canada

\section{RÉSUMÉ}

Cet article vise, d'une part, à faire le point sur l'état des connaissances actuelles en regard de ce qu'il est convenu d'appeler le trouble relatif au déficit de l'attention avec ou sans hyperactivité (TDA/H), et d'autre part, à examiner dans quelle mesure les élèves qui en sont atteints manifestent des comportements violents et s'il existe des types d'intervention aptes à contribuer à les réduire. Bien qu'il soit le trouble de comportement le plus commun diagnostiqué au cours de l'enfance, le TDA/H est plutôt mal connu et difficile à identifier et à diagnostiquer. Compte tenu de l'ampleur du phénomène, des conséquences observées sur le comportement des élèves qui en sont atteints, il s'avère important d'en connaître les symptômes et les conséquences, plus particulièrement en ce qui concerne les comportements violents. Les études tendent à montrer que les élèves ayant un TDA/H sont significativement plus nombreux que les autres à manifester des comportements colériques, agressifs, voire violents envers leurs pairs. De tels comportements entraînent des situations de conflit, d'impopularité et de rejet de la part de leurs pairs et plus d'attention négative de leur 
Le déficit de l'attention / hyperactivité (TDA/H)

et les comportements violents des jeunes en milieu scolaire : l'état de la question

enseignant. Les interventions qui font appel à des moyens qui aident les individus à contrôler leurs comportements apparaissent particulièrement appropriées pour amener les jeunes atteints de ce syndrome à réduire leurs comportements agressifs et violents et à développer leurs compétences sociales.

\section{ABSTRACT}

\section{Attention deficit disorder/ hyperactivity (add/H) and the violent behaviour of youth in the school setting: the current situation} Josée JUNEAU, Ph. D, Faculty of Education Sciences, Laval University, Louis-Philippe BOUCHER, Ph. D, Department of Education Sciences, University of Québec in Chicoutimi

This article attempts to clarify what is currently known about problems related to attention deficit disorder with or without hyperactivity. (ADD/H), to examine to what extent affected students manifest violent behaviour and whether there are interventions that could contribute to reducing these behaviours. Although it is the most common behavioural problem diagnosed in childhood, $\mathrm{ADD} / \mathrm{H}$ is poorly understood and difficult to identify and diagnose. Considering the scale of the phenomenon and the consequences observed on the behaviour of affected children, it is important to understand its symptoms and consequences, particularly in terms of violent behaviour. Studies tend to demonstrate that students with $\mathrm{ADD} / \mathrm{H}$ are significantly more likely than the others to manifest angry, aggressive, even violent behaviour towards their peers. Such behaviour leads to situations of conflict, unpopularity, rejection by peers, and more negative attention from their teachers. Interventions giving that provide students with ways of controlling their behaviour seem particularly appropriate for helping those with ADD to reduce their aggressive and violent behaviour and to develop social skills.

\section{RESUMEN}

El déficit de atención / hiperactividad (tda/h) y los comportamientos violentos de los jóvenes en el medio escolar: el estado de la cuestión Josée JUNEAU, Ph. D, Facultad de ciencias de la educación, Universidad Laval Louis-Philippe BOUCHER, Ph. D, Departamento de ciencias de la educación, Universidad de Québec en Chicoutimi

Este artículo pretende, por una parte, hacer balance de los conocimientos actuales sobre lo que se ha convenido en llamar el trastorno relativo al déficit de la atención con o sin hiperactividad (TDA/H), y por otra parte, examinar en qué medida los alumnos que sufren dicho déficit manifiestan comportamientos violentos y si existen tipos de intervención que pueden contribuir a su reducción. Aunque es el 
Le déficit de l'attention / hyperactivité (TDA/H)

et les comportements violents des jeunes en milieu scolaire : I'état de la question

trastorno del comportamiento más frecuentemente diagnosticado durante la infancia, el TDA/H es relativamente mal conocido y su identificación y diagnóstico es difícil. Teniendo en cuenta la magnitud del fenómeno y las consecuencias observadas sobre el comportamiento de los alumnos que son afectados, resulta importante conocer los síntomas y las consecuencias, particularmente en lo que concierne los comportamientos violentos. Los estudios tienden a demostrar que los alumnos que sufren de TDA/H presentan una tendencia importante en la demostración de comportamientos coléricos, agresivos, incluso violentos hacia sus pares. Dicho tipo de comportamiento provoca situaciones de conflicto, impopularidad y rechazo de la parte de los pares y atención negativa de la parte del maestro. Las intervenciones que utilizan medios que ayudan a los individuos a controlar sus comportamientos se presentan como particularmente apropiadas para ayudar a los jóvenes afectados por dicho síndrome a reducir los comportamientos agresivos y violentos y desarrollar sus habilidades sociales.

\section{Introduction}

Les enseignants sont souvent confrontés aux difficultés d'adaptation et d'apprentissage que vivent leurs élèves. Une de ces difficultés est due à ce qu'il est convenu d'appeler le trouble relatif au déficit de l'attention / hyperactivité (TDA/H). Bien qu'il soit le trouble de comportement le plus commun diagnostiqué au cours de l'enfance (Stewart, 1994; Sprafkin, Gadow \& Nolan, 2001), le TDA/H est plutôt mal connu et difficile à identifier et à diagnostiquer. Compte tenu de l'ampleur du phénomène, des conséquences observées sur le comportement des élèves qui en sont atteints, il s'avère important de le démystifier, c'est-à-dire d'en connaître les symptômes et les conséquences, plus particulièrement celles qui se rapportent aux comportements violents.

Après avoir décrit l'évolution du concept de TDA/H et en avoir défini les symptômes, nous abordons les difficultés reliées à son diagnostic, sa prévalence, ses effets évolutifs et ses conséquences sur le comportement des jeunes qui en sont atteints. Par la suite, nous nous demandons si les élèves atteints d'un TDA/H manifestent d'avantage de comportements violents envers leurs pairs et leurs enseignants que les autres élèves. Enfin, nous tentons d'identifier parmi les intervention de type éducatif celles qui apparaissent les plus aptes à aider les élèves atteints de ce syndrome à réduire leurs comportements violents et à développer leurs compétences sociales. 


\section{L'évolution du concept de TDA/H et son étiologie}

Le concept de TDA/H a une longue histoire, au cours de laquelle, il a pris différentes appellations. En 1902, Still (rapporté par Sandberg, 1996), prétend que le ralentissement cognitif observé chez des enfants ayant un problème d'inattention et d'hyperactivité est la conséquence d'un dommage au cerveau ou d'un retard de croissance. Ces spéculations conduisent à la création du concept de dysfonction cérébrale mineure (minimal brain dysfunction) signifiant qu'une déficience neurologique est à la base des difficultés d'apprentissage, du TDA/H et des perturbations émotionnelles. Cette théorie fut stimulée par une épidémie d'encéphalites, en 1912, aux États-Unis. À cette époque, un grand nombre d'enfants qui survécurent aux lésions cérébrales causées par cette maladie présentaient des problèmes d'inattention, d'hyperactivité et manifestaient des déficits sur le plan des habiletés cognitives, telles que la perception et la mémoire. Sur le plan social, ces enfants étaient perçus comme impulsifs, défiants et oppositionnels. Cependant, aucune de ces hypothèses n'a reçu d'appui empirique significatif (Barkley, 1990; Cantwell, 1996).

En 1968, l'American Psychiatric Association (L'APA) amorce des recherches relatives aux symptômes et au traitement du problème identifié, dans le DSM-II, sous l'appellation de réaction hyperkinésique de l'enfance. Ce problème se manifeste par une suractivité motrice ainsi que par un déficit de l'attention. Après plusieurs années de recherche et d'observation, l'APA remplace en 1980, dans le DSM-III, la catégorie réaction hyperkinésique de l'enfance par une autre comprenant deux types, à savoir : le déficit de l'attention avec hyperactivité et le déficit de l'attention sans hyperactivité. Cette catégorisation a l'inconvénient que, lorsque l'enfant ne présente pas de symptôme d'hyperactivité, aucun diagnostic n'est posé. Les seuls diagnostics émis se rapportent au déficit de l'attention avec hyperactivité qui est en quelque sorte associé aux comportements perturbateurs incluant les troubles des conduites, les troubles oppositionnels avec provocation et les difficultés d'apprentissage.

Pour réduire la confusion engendrée par ces deux types de trouble relatifs au déficit de l'attention, en 1987, l'APA modifie cette catégorie (le déficit de l'attention avec hyperactivité) pour la remplacer par l'hyperactivité avec déficit de l'attention. Ainsi, le symptôme principal réfère à l'hyperactivité. Cette décision prend sa source dans la conviction que ce type de difficulté est un trouble spécifique du développement et non un trouble de comportement. Cependant, comme l'impulsivité et l'hyperactivité ne sont pas toujours présentes dans les troubles relatifs au déficit de l'attention, le déficit de l'attention sans hyperactivité se trouve par le fait même éliminé.

Finalement, en 1994, l'APA modifie une autre fois cette catégorie par troubles relatifs au déficit de l'attention avec ou sans hyperactivité (TDA/H). La schizophrénie, les troubles affectifs et les retards mentaux sévères sont exclus du diagnostic. Cette nouvelle catégorisation prend en compte trois changements importants. Le premier concerne la division des symptômes en deux groupes (inattention et hyperactivité/impulsivité), le second, l'augmentation du nombre de symptômes afin de réduire la probabilité d'un mauvais diagnostic. Le troisième implique deux listes différentes de symptômes, une pour chacune des catégories. 
Le déficit de l'attention / hyperactivité (TDA/H)

et les comportements violents des jeunes en milieu scolaire: l'état de la question

Afin de ne pas alourdir le texte, l'appellation TDA/H est utilisée pour désigner les troubles relatifs au déficit de l'attention avec ou sans hyperactivité dans l'ensemble des études recensées, même si le déficit de l'attention n'était pas reconnu officiellement avant les années 1980. Dans les faits, tout indique qu'il s'agit essentiellement de la même clientèle.

\section{Les caractéristiques des enfants ayant un TDA/H}

Le TDA/H peut être diagnostiqué en fonction de trois catégories de symptômes : a) l'inattention et la distraction; b) l'hyperactivité; c) les difficultés à inhiber et l'impulsivité (APA, 1994). Précisons maintenant de quelle façon chacune de ces catégories de symptômes se manifeste.

L'inattention et la distraction des élèves aux prises avec un TDA/H se manifestent par une incapacité à se concentrer sur une tâche spécifique pendant une certaine période de temps, soit sur le plan scolaire, professionnel ou social.

Les comportements moteurs inadéquats en milieu scolaire constituent la caractéristique la plus évidente du TDA/H

\section{L'inattention et la distraction}

L'inattention et la distraction des élèves aux prises avec un TDA/H se manifestent par une incapacité à se concentrer sur une tâche spécifique pendant une certaine période de temps, soit sur le plan scolaire, professionnel ou social (APA, 1994; Cantwell, 1996; Hinshaw, Klein \& Abikoff, 1998; Zentall, Moon, Hall \& Grskovic, 2001). Le problème majeur de ces élèves est le manque d'attention soutenue dans les tâches répétitives (Barkley, 1998; Douglas, 1983; Landaw \& McAninch, 1993; Zentall et al., 2001). Ils sont plus distraits par les stimulations externes que les autres enfants (Zentall et al., 2001). Il n'est donc pas surprenant que les enseignants et les parents se plaignent que ces enfants n'écoutent pas, ne peuvent se concentrer, sont facilement distraits, ne finissent pas les tâches entreprises et égarent leurs choses. Ils tendent aussi à éviter les activités qui requièrent un effort intellectuel et/ou une attention soutenue, ou bien qui exigent de l'organisation. De plus, à partir d'observations réalisées par des parents, des enseignants et des pairs, il ressort que ces enfants sont plus timides et retirés sur le plan social que les autres enfants (Edelbrock, Costello \& Kessler, 1984; Lahey, Schaughency, Frame \& Strauss, 1985). Ce type de TDA/H serait, selon Scott (1987), sous-diagnostiqué.

\section{L’hyperactivité}

Les comportements moteurs inadéquats en milieu scolaire constituent la caractéristique la plus évidente du TDA/H (APA, 1994; Barkley, 1998; McKinney et al., 1993). Les élèves qui en sont atteints sont perçus comme « toujours sur le départ », ce qui se traduit par des comportements tels que se promener autour de la classe, ne pas tenir en place, se tortiller et s'agiter sur leur chaise. Ces enfants sont effectivement plus actifs, plus agités et plus impatients que les autres enfants (Rapport, DuPaul, Stoner \& Jones, 1986). De plus, ils sont plus sujets à recevoir un diagnostic des troubles des conduites (Hynd, Lorys, Semrud-Clikeman, Nieves, Huettner \& Lahey, 1991). La différence entre l'hyperactivité cliniquement significative et la suractivité, c'est que les comportements hyperactifs se manifestent indépendamment des situations (APA, 1994). 
Le déficit de l'attention / hyperactivité (TDA/H)

et les comportements violents des jeunes en milieu scolaire : l'état de la question

\section{L'impulsivité et la difficulté à inhiber}

Les comportements d'inattention, d'hyperactivité et d'impulsivité des enfants ayant un TDA/H seraient dus à un manque d'inhibition (Barkley, 1998; Newman \& Wallace, 1993; Oosterlaan, Logan \& Sergeant, 1998; Pennington \& Ozonoff, 1996). L'inhibition empêche habituellement les enfants d'émettre des comportements verbaux ou moteurs inadéquats dans un contexte social particulier (Barkley, 1998; McKinney, Montague \& Hocutt, 1993). Or, les enfants qui ont une faible capacité d'inhibition sont décrits comme répondant rapidement ou sans réfléchir, n'attendant pas leur tour dans les activités de groupe, faisant plusieurs erreurs d'inattention, prenant des risques inutiles et brisant négligemment leurs objets ou ceux des autres (Barkley, 1990; Zentall et al., 2001). Ils se mettent en colère très facilement et agissent sans penser aux conséquences de leurs gestes (Cantwell, 1996; Hinshaw et al., 1998). Ces enfants se caractérisent par un manque de contrôle de soi (Barkley, 1998; Hinshaw et al., 1998).

\section{La pose d'un diagnostic}

Il est souvent difficile à l'âge préscolaire de différencier les enfants ayant un TDA/H de ceux qui sont actifs et exubérants. Les symptômes spécifiques utilisés sont les crises de colère, les comportements obstinés, agressifs et intrépides pouvant causer des accidents, des blessures, des refus d'obtempérer et des problèmes de sommeil. Campbell (1990) établit qu'environ la moitié des enfants diagnostiqués hyperactifs à l'âge préscolaire sont reconnus comme ayant un TDA/H avant l'âge de neuf ans. Plus les symptômes sont sévères plus est élevée la probabilité que le déficit persiste.

Il n'existe pas de test diagnostique spécifique au TDA/H (Barkley, 1998; Cantwell, 1996; Desrosiers \& Royer, 1995; Sandberg, 1996). Les outils utilisés pour poser un diagnostic incluent des entrevues auprès des enfants et de leurs parents (compte rendu des symptômes et de leur impact sur la vie de l'enfant), des observations, des échelles de mesure du comportement, des examens physiques (visuels et auditifs) et neurologiques ainsi que des tests cognitifs. Finalement, en utilisant les critères du DSM-IV (1994), il est possible de diagnostiquer ce trouble, plus spécifiquement d'identifier un TDA/H à la condition que les comportements manifestés perdurent au delà de six mois.

Il y a des difficultés liées à l'identification des enfants ayant un TDA/H. La première se rapporte au contexte dans lequel évoluent les enfants durant les premières étapes de leur développement. Il est difficile, dû au fait que les jeunes enfants évoluent de façon plutôt solitaire, de déterminer si le comportement est normal ou s'il représente un problème transitoire ou persistant (Campbell, 1990). La deuxième concerne le diagnostic. En effet, celui-ci peut provoquer des perceptions négatives et ainsi amener des pressions excessives sur l'enfant et sur les parents pouvant même conduire au placement de l'enfant dans un environnement ségrégué (Fallen \& Umansky, 1985). La troisième difficulté a trait à la mesure des problèmes associés au 
Le déficit de l'attention / hyperactivité (TDA/H)

et les comportements violents des jeunes en milieu scolaire: l'état de la question

TDA/H et ainsi de différencier un type de problème (l'hyperactivité) d'un autre (les troubles des conduites). Cette difficulté s'avère plus grande quand l'enfant est de niveau préscolaire (Campbell, 1990).

\section{Les problèmes associés au TDA/H}

Selon le National Institutes of Health (1998), le TDA/H ne se présente rarement seul. Plus des deux tiers des élèves ayant un TDA/H manifeste effectivement au moins un autre type de problèmes, tels des problèmes de langage ou de communication, des difficultés d'apprentissage, des problèmes émotionnels ou des troubles des conduites. Un manque de savoir-faire social entraînerait, selon DuPaul \& Eckert (1997), des problèmes interpersonnels. Les résultats de la recherche tendent effectivement à confirmer que la corrélation entre le TDA/H et les troubles des conduites et les comportements oppositionnels avec provocation est relativement élevée, soit de 30 à 93 \% selon les études (Anderson, Williams, McGee \& Silva, 1987; Bird, Canino \& Rubio-Stipec, 1988; Bird, Gould \& Staghezza, 1993; Cohen, Velez, Brook \& Smith, 1989; Miranda \& Presentacion, 2000; Offord, Boyle \& Racine, 1989; Offord, Boyle \& Szatmari, 1987; Velez, Johnson \& Cohen, 1989).

\section{La prévalence relative du TDA/H}

Le taux de prévalence du TDA/H varie de façon importante en fonction du sexe et de l'âge des individus concernés. La proportion de garçons ayant un TDA/H serait, selon Stewart (1994), deux fois et demie plus élevées que celle des filles. Chez les enfants d'âge primaire, Hinshaw et ses collaborateurs (1998) évaluent sa fréquence à 3 ou $4 \%$ chez les garçons et à 1 ou $2 \%$ chez les filles. Lorsque l'on prend en compte les jeunes de 4 à 16 ans, sa fréquence augmente à $9 \%$ chez les garçons et à $3 \%$ chez les filles (Szatmari, Offord et Boyle, 1989). Selon deux autres études (Livingston, Dykman et Ackerman, 1990) et Breen et Altepeter, 1990), cette proportion serait d'une fille pour cinq garçons, dont $25 \%$ de ces derniers étant évalués hyperactifs et agressifs. Dans le DSM-IV (APA, 1994), on estime que cette proportion varie de quatre à neuf garçons pour une fille.

Cantwell (1996) et Sandberg (1996) font remarquer que les garçons démontrent typiquement plus de problèmes de comportement que les filles à l'école et sont identifiés plus souvent qu'elles comme ayant un TDA/H. Bien que les garçons et les filles ayant un TDA/H soient caractérisés par des relations sociales lacunaires, les filles se distinguent davantage par des déficits au plan de l'attention et de la cognition et par moins d'impulsivité, d'agressivité et de comportement antisociaux que les garçons (Barabasz \& Barabasz, 1996; Cantwell, 1996; Merrell \& Boelter, 2001; National Institutes of Health, 1998; Sandberg, 1996). Par ailleurs, elles démontrent plus de troubles de l'humeur, de l'affectivité et de l'émotivité (Barabasz \& Barabasz, 1996; Cantwell, 1996), ainsi que de meilleures compétences sociales (Merrell \& Boelter, 2001). 
Le déficit de l'attention / hyperactivité (TDA/H)

et les comportements violents des jeunes en milieu scolaire : l'état de la question

\section{Les effets évolutifs attribués au TDA/H}

Bien que la majorité des cas ne soit pas diagnostiquée avant l'âge scolaire, le TDA/H débuterait dans l'enfance (Sandberg, 1996). La suractivité, la distraction et les crises de colère chez les enfants d'âge préscolaire ressemblent aux symptômes du TDA/H chez les enfants plus âgés. Il est par conséquent difficile de faire la distinction entre les symptômes précurseurs de ce déficit et la manifestation passagère des comportements perturbateurs (Desrosiers \& Royer, 1995; Sandberg, 1996).

Selon diverses études, les symptômes du TDA/H observés chez les enfants persistent dans 50 à 80 \% des cas à l'adolescence (Barabasz \& Barabasz, 1996; Cantwell, 1996; DuPaul \& Eckert, 1998). Ces adolescents ont un piètre rendement scolaire, une faible estime de soi et des relations sociales déficientes. Ils sont aussi plus renfermés et moins communicatifs. Dans environ $25 \%$ des cas, les comportements antisociaux sont manifestes et font en sorte que le TDA/H devient un facteur de risque en regard de la criminalité à l'âge adulte (August \& Stewart, 1982; Cantwell, 1996; Miranda \& Presentacion, 2000).

\section{L'effet sur le rendement scolaire}

Les élèves ayant un TDA/H font montre généralement d'un faible rendement scolaire. Ils sont plus souvent placés en classe spéciale et ont davantage recours au tutorat comparativement aux autres élèves (August \& Garfinkel, 1989; Barkley, DuPaul \& Murray, 1990; Edelbrock et al., 1984; Kuehne, Kehle \& McMahan, 1987; Zental, 1990). Le sous-rendement scolaire apparaît associé à des troubles des conduites (Frick, Kamphaus, Lahey \& Loeber, 1991).

Selon DuPaul et Stoner (1994) et Hinshaw et ses collaborateurs (1998), les élèves ayant un TDA/H éprouvent des problèmes scolaires probablement en raison d'un manque d'engagement et d'une productivité non soutenue. Ces chercheurs ont observé, après avoir suivi des adolescents jusqu'à l'âge adulte, des taux significativement plus élevés de redoublement scolaire, de placement dans des classes spéciales et de décrochage chez ceux atteints d'un TDA/H que chez les autres. Ils ont constaté parallèlement que ces jeunes réalisent leurs travaux scolaires dans une proportion plus faible que leurs confrères. Cela peut expliquer, en partie, leur sous-rendement scolaire. Jusqu'à 80 \% d'entre eux auraient un faible rendement scolaire.

\section{Les compétences sociales inadéquates et les comportements violents}

Le risque pour les enfants ayant un TDA/H de développer des problèmes sociaux apparaît substantiellement élevé comparativement aux autres enfants (Merrell \& Boelter, 2001). Selon diverses études (Dykman \& Ackerman, 1991; Edelbrock et al., 1984; Nussbaum, Grant \& Roman, 1990), ces jeunes sont considérés sur le plan social 
Le déficit de l'attention / hyperactivité (TDA/H)

et les comportements violents des jeunes en milieu scolaire: l'état de la question

comme plus actifs, abusifs et agressifs que les autres. Ils sont également plus impulsifs dans les situations de conflit (Lahey et al., 1987; Stormont, 2001). Certains auteurs (Grenell, Glass \& Katz, 1987; Merrel \& Boelter, 2001; Nussbaum et al., 1990) prétendent que ces jeunes sont en quête de sensation forte d'où leur plaisir à être agressifs et dominateurs envers leurs pairs. Ils seraient aussi plus irresponsables et noninhibés (Lahey et al., 1985) que les jeunes de leur âge. Il ne faut pas s'étonner qu'en conséquence ils soient impopulaires auprès de leurs confrères (Carlson, Lahey, Frame, Walker \& Hynd, 1986; Edelbrock et al., 1984; Hinshaw et al., 1998; Lahey et al., 1985). Cette impopularité serait due au fait qu'ils sont plus souvent en conflit avec les autres (Wacks \& Gilman, 1996). Selon Miranda et Presentacion (2000) et Sprafkin, gadow et Nolan (2001), il existe un lien étroit et significatif entre le TDA/H et les relations conflictuelles, les comportements agressifs et délinquants.

Bien que ces jeunes ne diffèrent pas significativement de leurs pairs en ce qui concerne la maitrise de stratégies pour initier les relations sociales, ils sont, par contre, moins amicaux et efficaces pour maintenir ces relations (Grenell et al., 1987; Merrell \& Boelter, 2001; Sprafkin, Gadow et Nolan., 2001). En fait, ils ont de piètres compétences sociales plutôt qu'un manque d'habiletés. Ces faiblesses se manifestent principalement par des comportements antisociaux, tels l'intrusion, l'irritabilité et la colère (Merrell \& Boelter, 2001). Ainsi, la corrélation entre le TDA/H et les comportements antisociaux est considérée comme significativement élevée par plusieurs chercheurs (Berry, Shaywitz \& Shaywitz, 1985; Lahey et al., 1987; Merrel \& Boelter, 2001; Stormont, 2001). En fait, un manque de contrôle de soi et une gestion déficiente du comportement additionnés à un niveau excessif d'irritabilité et de comportements colériques seraient à la source des comportements antisociaux manifestés par ces jeunes. Merrell et Boelter (2001) observent que lorsque les symptômes du TDA/H augmentent, les comportements sociaux positifs diminuent et inversement, d'où la relation étroite et significative entre le TDA/H et les comportements sociaux inadéquats.

Stormont (2001) affirme que l'un des phénomènes les plus envahissants chez les enfants ayant un TDA/H est le comportement d'agression qui est fortement corrélé au rejet par les pairs. Ils sont décrits par ces derniers comme amorçant toujours les batailles et les prises de bec. Toujours selon cette étude, la majorité de ces jeunes (62\%) présentent des déficits sérieux au plan social comparativement aux autres jeunes (12\%). Ils manifestent, entre autres, un manque de maitrise des comportements sociaux acceptables. Par exemple, dans les situations de conflits, ils se comportent de façon plus agressive et moins amicale tout en prétendant que ce sont eux les meilleurs. Ils sont également plus malhonnêtes (tricheurs), plus critiques envers leurs pairs et peu communicatifs.

Bien qu'aucune recherche portant directement sur les comportements agressifs des élèves ayant un TDA/H envers leur enseignant n'ait été identifiée, Stormont (2001) fait observer, qu'en raison de l'ensemble de leurs comportements inadéquats, les enseignants ont tendance à adopter une attitude plus négative envers ces élèves qu'envers les autres élèves. Ils reçoivent donc ainsi plus d'attention négative de la part de leur enseignant. 


\section{Les interventions favorisant un contrôle des comportements violents}

Dans le but de réduire l'incidence des comportements d'agression des jeunes atteints d'un TDA/H et/ou d'augmenter leurs compétences sociales, divers types d'interventions ont été conçues et expérimentés. Parmi les interventions éducatives, c'est-à-dire celles visant à donner plus de pouvoir à la personne sur son comportement (ce qui implique l'exclusion de toute médication), nous présentons celles qui apparaissent les plus prometteuses.

Zentall et ses collaborateurs (2001) suggèrent qu'au lieu de s'attaquer aux comportements inappropriés des jeunes atteints d'un TDA/H, les intervenants devraient privilégier avant les façons de faire visant à augmenter leur motivation et leur estime de soi. Pour ce faire, ils proposent de placer les jeunes dans des situations leur permettant d'être aidants ou leaders. Ce type d'intervention tend à augmenter leurs compétences sociales puisqu'ils travaillent et collaborent mieux quand ils sont en charge d'un projet qui stimule leur motivation et renforce leur estime de soi.

Pour faire diminuer des manifestations comportementales négatives comme l'intrusion, l'irritabilité et les comportements explosifs qui constituent des facteurs explicatifs des comportements agressifs, Merrell \& Boelter (2001) recommandent des interventions faisant appel à des procédés d'auto-contrôle. Compte tenu que les enfants ayant un TDA/H se caractérisent davantage par un manque de contrôle de soi dû à des difficultés d'inhibition qu'à un manque d'attention, les procédés d'autocontrôle, tels l'autoverbalisation, l'autoenregistrement, l'autoévaluation et l'autorenforcement sont, selon Berkeley (1997), particulièrement efficaces auprès de ce type de clientèle. Ces procédés, découlant d'une approche cognitivo-béhaviorale, ont justement pour but de les amener à assumer la direction de leurs comportements (Bandura, 1980; Staats, 1986),.

Afin d'améliorer la concentration et la réflexion des élèves ayant un TDA/H en situation d'apprentissage ou en situation sociale, Miranda et Presentacion (2000) ont utilisé divers procédés, dont l'auto-instruction (arrête-pense), le modelage et les contingences comportementales. De plus, ils ont fait appel à une technique spécifique pour le contrôle de la colère fondée sur le fait que, dans des situations sociales, les élèves ayant un TDA/H et de l'agressivité répondent immédiatement avec violence. Ainsi, une des premières choses à faire pour diminuer de tels comportements est de leur faire identifier les facteurs relatifs à la manifestation de la colère, de leur apprendre à se détendre dans ces situations et à contrôler leur colère. Ces interventions s'avèrent significativement positives en ce qui a trait à la réduction des symptômes du TDA/H et à la diminution des comportements antisociaux, dont les comportements agressifs.

Dans le but de prévenir les comportements violents chez les jeunes atteints d'un TDA/H, Shure (1999) a fait appel à un type d'intervention connue sous le nom de Interpersonal Cognitive Problem-Solving (ICPS Skills). Il vise à leur apprendre à penser avant d'agir. Les élèves ayant bénéficié d'un tel type d'intervention précoce peuvent effectivement apprendre à contrôler leur colère en découvrant de nouvelles 
Le déficit de l'attention / hyperactivité (TDA/H)

et les comportements violents des jeunes en milieu scolaire: l'état de la question

façons de l'exprimer et à reconnaître les conséquences de leurs gestes, réduisant ainsi l'émergence de comportements agressifs et plus tard de délinquance (Shure, 1999). Se situant dans une même courant de pensée, Stormont (2001) suggère des interventions visant à développer diverses habiletés cognitives dont apprendre à écouter, à attendre son tour dans une conversation, donner des compliments, exprimer sa colère de façon appropriée, suivre des règles, assister les autres, initier une conversation, joindre un groupe, et demander de l'assistance de façon appropriée.

\section{Conclusion}

Le syndrome du TDA/H s'est précisé au cours des vingt dernières années. Il prend en compte deux grandes catégories de symptômes, à savoir le déficit de l'attention et l'hyperactivité, ainsi que les types prédominants. Les symptômes sont suffisamment précis et observables pour permettre aux intervenants scolaires d'identifier les élèves qui sont susceptibles d'avoir un TDA/H. La pose d'un diagnostic officiel demeure toutefois une entreprise exigeante compte tenu de la quantité des outils devant être utilisés et la nécessaire collaboration de plusieurs personnes.

Les données de recherche relatives à l'ampleur et les manifestations du phénomène, selon les catégories de personnes, sont pour le moins imprécises. On constate des écarts très importants d'une étude à l'autre. Il y aurait deux à neuf fois plus de garçons que de filles qui en sont atteints.

Le syndrome du TDA/H ne disparaît pas à l'adolescence, mais persiste dans la grande majorité des cas. Ses conséquences peuvent être très handicapantes pour les jeunes qui ne reçoivent pas de traitement approprié, telles une faible estime de soi, des comportements antisociaux, de délinquance et un faible rendement scolaire. En général, les enfants à risque qui reçoivent un programme éducatif précoce sont moins susceptibles d'être placés en éducation spéciale, de demeurer au même niveau, de manifester des comportements délinquants.

Les élèves ayant un TDA/H sont significativement plus nombreux que les autres à manifester des comportements colériques, agressifs, voire violents envers leurs pairs. De tels comportements entraînent des situations de conflit, d'impopularité et de rejet de la part de leurs confrères et plus d'attention négative de la part de leur enseignant ou enseignante. Ils seraient dus, d'une part, à des déficits importants dans leurs compétences sociales, dont celles d'établir et de maintenir des relations positives avec les autres, et d'autre part, à un manque de contrôle de soi et de gestion de comportement. Les interventions qui apparaissent les plus efficaces pour aider les élèves ayant un TDA/H à réduire leurs comportements antisociaux et à augmenter leurs compétences sociales sont celles qui les amènent à s'arrêter et à réfléchir avant d'agir ou qui leur apprennent à contrôler leurs comportements à l'aide de procédés d'autocontrôle.

Il y a, par conséquent, de l'espoir pour les enfants qui sont atteints d'un TDA/H à la condition qu'ils puisent bénéficier d'une intervention précoce reposant sur des approches et procédés qui leur permettent de développer des compétences sociales 
et d'acquérir un meilleur contrôle sur leurs comportements. Les parents et les enseignants de ces enfants ou de ces élèves doivent avoir la possibilité de s'initier à ces moyens afin de pouvoir les aider à développer leurs compétences sociales et ainsi à fonctionner le plus adéquatement possible en société.

\section{Références bibliographiques}

American Psychiatric Association (1968). Diagnostic and statistical manual of mental disorders. Third edition (DSMII). Paris: Masson.

American Psychiatric Association (1980). Diagnostic and statistical manual of mental disorders. Third edition (DSMIII). Paris: Masson.

American Psychiatric Association (1987). Diagnostic and statistical manual of mental disorders. Third edition, revised (DSMIII-R). Paris: Masson.

American Psychiatric Association (1994). Diagnostic and statistical manual of mental disorders. Fourth edition (DSMIV). Paris: Masson.

Anderson, J. C., Williams, S., McGee, R., \& Silva, P. A. (1987). DSM-III disorders in preadolescent children. Archives General Psychiatry, 44, pp. 69-76.

August, G. J., \& Garfinkel, B. Behavioral and cognitive subtypes of ADHD (1989). Journal of the American Academy of Child and Adolescent Psychiatry, 28, pp. 739-748.

August, G. J., \& Stewart, M. A. (1982). Is there a syndrome of pure hyperactivity? British Journal of Psychiatry, 140, pp. 305-311.

Bandura, A. (1980). L'apprentissage social. Bruxelle: Mardaga.

Barkley, R. A. (1990). Attention deficit hyperactivity disorder: A handbook for diagnosis and treatment. New York: Gilford Press.

Barkley, R. A. (1997). ADHD and the nature of self control. New York: Guilford Press.

Barkley, R. A. (1998). Attention deficit hyperactivity disorder: A handbook for diagnosis and treatment. Second edition. New York: Guilford Press.

Barkley, R. A., DuPaul, G., \& McMurray, M. (1990). Comprehensive evaluation of attention deficit disorder with and without hyperactivity as defined by research criteria. Journal of Consulting and Clinical Psychology, 58, pp. 775-789.

Barabasz, M., \& Barabasz, A. (1996). Attention deficit disorder: Diagnosis, etiology and treatment. Child Study Journal, 26(1), pp. 1-37.

Berry, C. A., Shaywitz, S. E., \& Shaywitz, B. A. (1985). Girls with ADD: A silent minority? A report on behavioral and cognitive characteristics. Pediatrics, 76, pp. 801-809. 
Le déficit de l'attention / hyperactivité (TDA/H)

et les comportements violents des jeunes en milieu scolaire : l'état de la question

Bird, H. R., Canino, G., \& Rubio-Stipec, M. (1988). Estimates of the prevalence of childhood maladjustment in a community survey in Puerto Rico. Archives General of Psychiatry, 45, pp. 1120-1126.

Bird, H. R., Gould, M. S., \& Staghezza, B. M. (1993). Patterns of diagnostic comorbidity in a community sample of children aged 9 through 16 years. Journal American Academy Child and Adolescents Psychiatry, 32, pp. 361-368.

Breen, M. J., \& Alterpeter, T. S. (1990). The home situations questionnaire and the school situations questionnaire: Normative data and an evaluation of psychometric properties. Journal of Psychoeducational Assessment, 7, pp. 312-322.

Campbell, S. B. (1990). Psychiatric disorder in preschool children. New York: Gilford Press.

Cantwell, D. P. (1996). Attention deficit disorder: A review of the past 10 years. Journal of the American Academy of Child and Adolescent Psychiatry, 35(8), pp. 978-987.

Carlson, C., Lahey, B., Frame, C., Walker, J., \& Hynd, G. (1986). Sociometric status of clinic-referred children with ADD with and without hyperactivity. Journal of Abnormal Child Psychology, 15, pp. 537-547.

Cohen, P., Velez, C. N., Brook, J., \& Smith, J. (1989). Mechanisms of the relation between perinatal problems, early childhood illness, and psychopathology in late childhood and adolescence. Child Development, 60, pp. 701-709.

Desrosiers, K., \& Royer, E. (1995). Les troubles de l'attention avec hyperactivité: Une synthèse des connaissances à l'intention des enseignants. Centre de recherche et d'intervention sur la réussite scolaire, Université Laval, Québec.

Douglas, V. I. (1983). Attention and cognitive problems, dans Developmental neuropsychiatry, sous la direction de M. Rutter (Eds). New York: Gilford Press, pp. 280-329.

DuPaul, G. J., \& Eckert, T. L. (1997). The effects of school-based interventions for attention deficit hyperactivity disorder: A meta-analysis. School Psychology Review, 26(1), pp. 5-27.

DuPaul, G. J., \& Eckert, T. L. (1998). Academic interventions for students with attention-deficit/hyperactivity disorder: A review of the literature. Reading and Writing Quarterly: Overcoming Learning Difficulties, 14(1), pp. 59-82.

DuPaul, G. J., \& Stoner, G. (1994). ADHD in the school: Assessment and intervention strategies. New York: Gilford Press.

Dykerman, R., \& Ackerman, P. (1991). ADD and specific reading disability: Separate but often overlapping disorders. Journal of learning Disabilities, 24, pp. 96-103.

Edelbrock, C., Costello, A., \& Kessler, M. (1984). Empirical corroboration of attention deficit disorder. Journal of the American Academy of Child Psychiatry, 23, pp. 285-290. 
Le déficit de l'attention / hyperactivité (TDA/H)

et les comportements violents des jeunes en milieu scolaire : l'état de la question

Fallen, N. H., \& Umansky, W. C. (1985). Young children with special needs. Columbus, OH: Merrill.

Frick, P. J., Kamphaus, R.W., Lahey, B.B., \& Loeber, R. (1991). Academic underachievement and the disruptive behavior disorders. Journal of Consulting Child Psychology, 21, pp. 273-305.

Grenell, M., Glass, C., \& Katz, K. (1987). Hyperactive children and peer interaction: Knowledge and rendement of social skills. Journal of Abnormal Child Psychology, 15, pp. 1-13.

Hinshaw, S. P., Klein, R. G., \& Abikoff, H. (1998). Childhood attention deficit hyperactivity disorder: Nonpharmacological and combination treatments, dans A guide to treatments that work, New York: Oxford University Press, pp. 26-41.

Hynd, G. W., Lorys, A. R., Semrud-Clikeman, M., Nieves, N., Huettner, M., \& Lahey, B. B. (1991). ADD without hyperactivity and neurocognitives syndrome. Journal of Child Neurology, 6, pp. 37-41.

Kuehne, C., Kehle, T., \& McMahan, W. (1987). Differences between children with ADD, children with SLD, and normal children. Journal of School Psychology, 25, pp. 161-166.

Lahey, B. B., Schaughency, B. B., Frame, C. L., \& Strauss, C. C. (1985). Teacher ratings of attention problems in children experimentally classified as exhibiting ADD with and without hyperactivity. Journal of the American Academy of Child Psychiatry, 24, pp. 613-616.

Lahey, B. B., Schaughency, E., Hynd, G., Carlson, C., \& Nieves, N. (1987). Attention deficit disorder with and without hyperactivity: Comparison of behavioral characteristics of clinic-referred children. Journal of the American Academy of Child Psychiatry, 26, 1987, pp. 718-723.

Landaw, S., \& McAninch, C. (1993). Young children with attention deficits. Young children, pp. 49-57.

Lazar, I., \& Darlington, R. (1982). Lasting effects of early education. Monographs of the Society for Research in Child Development, 47, pp. 47-59.

Livingston, R. L., Dykman, R. A., \& Ackerman, P. T. (1990). The frequency and significance of additional self-reported psychiatric diagnoses in children with ADD. Journal of Abnormal Child Psychology, 18, pp. 465-478.

McKinney, J. D., Montague, M., \& Hocutt, A. M. (1993). Synthesis of research on the assessment and identification of students with attention deficit disorders. Washington, D.C: Special Education Programs.

Merrell, K.W., \& Boelter, E. (2001). An investigation of relationships between social behavior and ADHD in children and youth: Construct validity of the home and community social behavior scales. Journal of Emotional and Behavioral disorders, 9(4), pp. 260-269. 
Le déficit de l'attention / hyperactivité (TDA/H)

et les comportements violents des jeunes en milieu scolaire : l'état de la question

Miranda, A., \& Presentacion, M. J. (2000). Efficacy of cognitive-behavioral therapy in the treatment of children with ADHD, with and without aggressiveness. Psychology in the schools, 37(2), pp. 169-182.

National Institutes of Health. (1998). Diagnosis and treatment of attention deficit hyperactivity disorder (ADHD). Consensus development conference statement.

Newman, J. P., \& Wallace, J. F. (1993). Diverse pathways to deficient self-regulation: Implications for disinhibitory psychopathology in children. Clinical Psychology Review, 13, pp. 699-720.

Nussbaum, N. L., Grant, M. L., \& Roman, M. J. (1990). ADD and the mediating effect of age on academic and behavioral variables. Journal of Developmental and Behavior Pediatrics, 11, pp. 22-26.

Offord, D. R., Boyle, M. H., \& Racine, Y. (1989). Ontario child health study: Correlates of disorder. Journal American Academy Child and Adolescent Psychiatry, 28, pp. 856-860.

Offord, D. R., Boyle, M. H., \& Szatmari, P. (1987). Ontario child health study II: Six month prevalence of disorder and rates of service utilization. Archives General Psychiatry, 44, pp. 832-836.

Oosterlaan, J. L., Logan, G. D., \& Sergeant, J. A. (1998). Response inhibition in $\mathrm{AD} / \mathrm{HD}, \mathrm{CD}$, comorbid $\mathrm{AD} / \mathrm{HD}+\mathrm{CD}$, anxious, and control children: A meta-analysis of studies with the stop task. Journal of Child Psychology and Psychiatry and Allied Disciplines, 39(3), pp. 411-425.

Pennington, B. F., \& Ozonoff, S. (1996). Executive functions and developmental psychopathology. Journal of Child Psychology and Psychiatry, 37, pp. 51-87.

Rapport, M. D., DuPaul, G. J., Stoner, G., \& Jones, J. I. (1986). Comparing classroom and clinic measures of attention deficit disorder: Differential, idiosyncratic, and dose-response effects of methylphenidate. Journal of Consulting and Clinical Psychology, 54, pp. 334-341.

Sandberg, S. (1996). Hyperkinetic or attention deficit disorder. British Journal of Psychiatry, 169 (1), pp. 10-17.

Scott, M. (1987). Attention deficit disorder. Washington, D. C. Office of educational research and improvement.

Shure, M. B. (1999). Preventing violence the problem-solving way. Juvenile justice bulletin. Office of juvénile justice and delinquant prevention. Washington, DC, $13 \mathrm{p}$.

Sprafkin, J., Gadow, K.D., \& Nolan, E.E. (2001). The utility of a DSM-IV-referenced screening instrument for attention-deficit hyperactivity disorder. Journal of Emotional and Behavioral Disorders, 9(3), pp. 182-191.

Staat, A. W. (1986). Behaviorisme social. Traduction de A. Leduc \& R. Beausoleil. Brossard (Québec) : Behaviora. 
Stewart, G. A. (1994). External validity of attention deficit disorder without hyperactivity: A review of the evidence. Canadian Journal of School Psychology, 10(1), pp. 15-25.

Stormont, M. (2001). Social outcomes of children with ADHD: Contributing factors and implications for practice. Psychology in the Schools, 38(6), pp. 521-531.

Szatmari, P., Offord, D. R., \& Boyle, M. H. (1989). Ontario child health study: Prevalence of attention deficit disorder with hyperactivity. Journal of Child Psychology Psychiatry and Allied Disciplines, 30, pp. 219-230.

Velez, C. N., Johnson, J., \& Cohen, P. (1989). A longitudinal analysis of selected risk factors for childhood psychopathology. Journal American Academy Children and Adolescent Psychiatry, 28, pp. 861-864.

Wacks, J. M., \& Gilman, D. A. (1996). Follow-up study on the adjustment of ADHD Adolescence.

Zentall, S. S. (1990). Fact-retrieval automatization and math problem solving by LD, ADD, and normal adolescents. Journal of Educational Psychology, 82, pp. 856-865.

Zentall, S. S., Moon, S., Hall, A., \& Grskovic, J. (2001). Learning and motivational characteristics of boys with $\mathrm{AD} / \mathrm{HD}$ and/or giftedness. Exceptional Children, 67(4), pp. 499-519. 